\title{
Editorial
}

\section{Interpretation of thyroid function tests: a challenge to the clinician?}

\author{
Dimuthu T Muthukuda, Uditha Bulugahapitiya \\ Sri Lanka Journal of Diabetes, Endocrinology and Metabolism 2013; 3: 1-3
}

The array of medical professionals dealing with thyroid related problems is vast. The burden of thyroid illnesses is such that it mandates almost every clinician to excel in the interpretation of thyroid function tests (TFT). This is often straightforward, but could be confusing and complex at times, resulting in mismanagement. The purpose of this editorial is to reiterate the need to be conversant in interpreting unusual TFT patterns; hence this is by no means a comprehensive review of the topic.

A sound understanding of the pathophysiology of the hypothalamic-pituitary-thyroid (HPT) axis is the key to accurate interpretation of TFTs. In conformity with this, the basic principles should be extrapolated to analyse the deviations, which are manifold. The clinical manifestations of thyroidal illness further confound this, as often the symptoms and signs could be very subtle and nonspecific in nature.

Various pre-analytical and analytical factors could lead to alterations in the TFT pattern giving rise to unconventionalities (1).

TSH, free T4 and free T3 are the most commonly performed tests (2). The negative log linear pattern results in broader reciprocal fluctuations of the TSH in relation to the free hormones, hence TSH being more indicative of thyroidal illness where the free hormones remain normal, assuming that the HPT axis is intact (3). Highly sensitive TSH assays have a detection limit less than $0.01 \mathrm{mU} / \mathrm{l}$ (4, 5). Therefore TSH is considered sufficiently sensitive in both primary hyper- and hypothyroidism as the sole parameter. However, TSH alone could be misleading in certain instances such as central hypothyroidism where TSH could be inappropriately normal.

Virtually all of thyroid hormone is bound to TBG (thyroxine binding globulin), transthyretin (thyroxinebinding prealbumin) or albumin. In conditions which can alter the concentrations of binding proteins, the free and total hormone concentrations may not be concordant. Most laboratories in the present era have overcome this problem of euthyroid hyperthyroxinaemia by measuring the free hormone levels. Familial dysalbuminemic hyperthyroxinemia is a genetic disorder, characterized by the presence of mutant albumin molecules that show altered binding to T4 and T3. Despite the use of free T4, this condition can result in a falsely high value unless special techniques are used (3).

Concomitant use of various drugs can alter the TFTs; dopamine and steroids are known to cause a suppression of TSH (1). Amiodarone is well-known to affect the thyroid gland in various ways due to its inherent properties. It also inhibits T4 conversion to T3. As a result, patients treated with amiodarone who do not develop hypo- or hyperthyroidism often have low T3 concentrations, while $\mathrm{T} 4$ is raised to just above the upper limit of the normal (6). Propranolol and related drugs, besides propylthiouracil are also known to cause a similar pattern. Another characteristic pitfall is the effect of heparin. Free thyroid hormones could be elevated suggesting hyperthyroidism. Heparin induces the endothelial lipoprotein lipase activity liberating free fatty acids to the circulation thereby displacing the free thyroid hormones from TBG $(3,7)$. This can be further aggravated by delaying the sample analysis and is more significant when the triglyceride levels are high. Total hormones become valuable in this instance.

Interpretation of the TFTs in the presence of nonthyroidal illness is a challenge. If possible the assessment of the thyroid status should be deferred till the patient recovers. However, at times it becomes vital to exclude a thyroidal problem in the acute setting. The TFT pattern depends on the severity and stage of the underling illness, not to mention the confounding effects of drugs (1). Hence it is not straightforward to identify a constant pattern in non-thyroidal illness. The best indicator, if desired to identify a thyroidal illness in this setting is TSH.

Pregnancy is associated with various changes in the TFTs and interpretation has to be done very cautiously with trimester specific reference ranges, which is a topic in its own right. The thyroid hormone axis in extremes of age is another consideration. Due to the maturing process of the hormone axis, high TSH and free hormone levels are seen in the neonatal period (1). In the elderly the TSH levels could be slightly high although a uniform reference range is utilized across all age groups in adults. The importance of age-based normal ranges for TSH was illustrated in the National Health and Nutrition Examination Survey III (NHANES III), which showed an age-related 
shift towards higher TSH concentrations in older patients, even when the high risk population was excluded (8).

Poor compliance should not be underrated as a cause of erroneous results. This is based on the half-lives of TSH and thyroid hormones which differ to a great extent. TSH may be discordant with free hormones with intermittent intake of thyroxine. Therefore it is prudent to elucidate the accurate background information prior to final decision making.

High free hormones and non-suppressed TSH, either inappropriately normal or high, is another classic constellation that should not be ignored. Having excluded technical problems of the assay and use of interfering drugs, the differential diagnosis is between resistance to thyroid hormones (RTH) and TSH secreting pituitary tumour (TSHoma) (3). Both conditions are rare and the clinical features may not be discriminatory, although a positive family history and concomitant evidence of a pituitary macroadenoma may point towards a diagnosis of RTH and TSHoma respectively. Other tests are needed to differentiate as imaging could be deceptive if done prior to biochemical confirmation. Incidentalomas could co-exist with RTH perplexing the picture; further, microadenonas may not be visualized on magnetic resonance scans unless dynamic images are obtained. Tissue markers of $\mathrm{TH}$ action including serum sex hormone-binding globulin concentrations (SHBG) are generally high with TSHoma, whereas it is normal in RTH. The serum alphasubunit ( $\alpha \mathrm{SU}$ ) and $\alpha$ SU/TSH molar ratio are normal in $\mathrm{RTH}$, but often high in TSHoma due to co-secretion. The serum TSH concentration generally increases in response to TRH in RTH, but absent or blunted in TSHoma due to the autonomy of the thyrotrophs. In addition, patients with RTH are more likely to have a fall in serum TSH in response to T3 $(3,9,10)$. Indeed, no biochemical marker or dynamic test is solely pathognomonic in the diagnosis of these rare conditions. Genetic testing will aid the diagnostic process. If not properly diagnosed, inadvertent surgeries could take place, which was the case in the past with the old TSH assays (11).

Apparent deviations from the normal thyroid physiology due to analytical factors are also worth mentioning. Falsely high TSH, discordant with the thyroid status could ensue due to technical faults of the assay (12). In this scenario, we need to exclude assay interference. This is known to occur with heterophille antibodies or human anti animal antibodies (HAAs), which could cross react with the antibodies used in the assay (3). These are antibodies to animal antigens, which were acquired, as a result of previous infections, exposure to medications containing similar antigens or occupational exposure. Certain poly-specific antibodies, i.e. rheumatoid factor, can also cross react with the assay and give rise to fake results. These antibodies can cross-link the capture and detection antibodies of the sandwich type TSH assay, thereby giving rise to positive interference. Similarly a negative interference can result in a falsely low TSH. On clinical suspicion, the test could be repeated using a different assay with different antibodies. Sample dilution and addition of blocking or precipitating agents are certain techniques that exist in clinical practice to overcome this technical error (12). Free thyroid hormones are no exception to this problem and should be considered if a discrepancy exists in the clinical context. In addition, patients with an autoimmune tendency can harbour antibodies to thyroid hormones which can give rise to artefacts (1). The vast array of possible technical challenges concludes that unless extensively sought, this phenomenon could escape detection $(12,13)$.

Which tests to perform remains a challenge with the complexity of the TFTs. It is not practical to give a unifying answer to this question simply because of the broader spectrum of deviations encountered. It is noteworthy that the assays for free T3 are less well validated than those for free T4, showing a greater degree of variation (4). However the utility of this becomes mandatory as in the case of normal free T4 in a hyperthyroid patient indicating T3-toxicosis.

This account exemplifies some of the pitfalls encountered in the interpretation of TFTs. It is highly unlikely that any clinician is exempted from this challenge in routine practice. An alerted mind-set is obligatory to channel the patient for expert advice. History and clinical examination are vital components and should not be underestimated as TFTs should always be interpreted in the clinical context. Isolated reports do not warrant management decisions and at times could be detrimental to the patient. It is also noteworthy to mention the importance of good communication between the clinician and the laboratory.

The vastness of the subject is beyond the scope of this account. The mere flavour of the diversity of the thyroid function tests reiterates the importance of having a vigilant mind to the more or less inevitable encounter of an unusual thyroid function test pattern.

- Joint Editors

\section{References}

1. Carolyn Fedler. Laboratory tests of thyroid function: pitfalls in interpretation. CME 2006; 24(7): 386-90.

2. Beckett GJ, Toft AD. First-line thyroid function tests TSH alone is not enough. Clinical Endocrinology 2003; 58: 20-21.

3. Gurnell M, Halsall DJ, ChatterjeeVK. What Should be Done When Thyroid Function Tests Do Not Make Sense? Clin Endocrinol 2011; 74(6):673-8.

4. Bahn RS, Burch HB, Cooper DS, Garber JR, Greenlee MC, et al. Hyperthyroidism and Other Causes of Thyrotoxicosis: 
Management Guidelines of the American Thyroid Association and American Association of Clinical Endocrinologists. Thyroid 2011; 21(6): 593-646.

5. Lynn R. Witherspoon. Clinical Utility of Sensitive TSH. Lab Med 2005; 36(11): 711-15.

6. Cohen-Lehman J, Dahl P, Danzi, S, et al. Effects of amiodarone therapy on thyroid function. Nature Reviews Endocrinology 2010; 6: 34-41.

7. Jaume JC, Mendel CM, Frost PH, et al. Extremely low doses of heparin release lipase activity into the plasma and can thereby cause artifactual elevations in the serum-free thyroxine concentration as measured by equilibrium dialysis. Thyroid 1996; 6: 79.

8. Surks MI, Hollowell JG. Age-specific distribution of serum thyrotropin and antithyroid antibodies in the US population: implications for the prevalence of subclinical hypothyroidism. J Clin Endocrinol Metab 2007; 92: 4575.

9. Brucker-Davis F, Oldfield EH, Skarulis MC, et al. Thyrotropin-secreting pituitary tumors: diagnostic criteria, thyroid hormone sensitivity, and treatment outcome in 25 patients followed at the National Institute of Health. J Clin Endocrinol Metab 1999; 84: 476.

10. Kienitz T, Quinkler M, Strasburger CJ, Ventz M. Longterm management in five cases of TSH-secreting pituitary adenomas: a single center study and review of the literature. European Journal of Endocrinology 2007; 157: 39-46.

11. Socin HV, Chanson P, Delemer B, et al. The changing spectrum of TSH-secreting pituitary adenomas: diagnosis and management in 43 patients. Eur J Endocrinol 2003; 148: 433.

12. Ross HA, Menheere PPCA, Thomas CMG, Mudde AH, Kouwenberg M, Wolffenbuttel BHR. Interference from heterophilic antibodies in seven current TSH assays. Annals of Clinical Biochemistry 2008; 45: 616-18.

13. Ismail AAA, Barth JH, Walker PL, Cawood M. Interference in immunoassay is an underestimated problem. Ann Clin Biochem 2002; 39: 366-73. 